\title{
The Role of Helicobacter Pylori Infection in Idiopathic Thrombocytopenic Purpura in Children
}

\author{
Al Zahraa Elsayed Ahmed, Ashraf Mahmoud Radwan, Heba Latif Rizk* \\ Department of Pediatric, Faculty of Medicine, Sohag University \\ *Corresponding author: Heba Latif Rizk, Mobile: (+20) 01283523294, E-Mail: hebalatif90@ gmail.com
}

\begin{abstract}
Background: Many studies had found obvious relation between helicobacter pylori infection and chronic idiopathic thrombocytopenic purpura; several studies have reported remission of immune thrombocytopenic purpura (ITP) after eradication of a coexistent Helicobacter pylori infection in adults, data in children are limited.

Objective: To detect the association of H. pylori infection and idiopathic thrombocytopenic purpura in children and the effect of eradication of the infection on platelet count in these patients.

Patients and methods: This study was conducted on 83 chronic idiopathic thrombocytopenic purpura children with age range from 3 to 13 years. They were 46 males 37 females. Also the study included 104 healthy individual with the same range of age. They were 58 males and 46 females as a control group. This was between October 2018 and September 2019 in the Pediatric Hematology and GIT Clinics or inpatients wards of Pediatric Department.

Results: Clinical benefit occurred in 31 cases of the 83 chronic ITP (cITP) patients screened; nevertheless, we have a simple, inexpensive, non-invasive screening method; the adverse events of eradication therapy are negligible, together with an affordable cost, all uncommon attributes among the specific treatments currently available for cITP; therefore it is worthwhile to test for and treat $\mathrm{H}$. pylori infection.

Conclusion: Our data showed a prevalence of $\mathrm{H}$. pylori infection in cITP pediatrics that was similar to other studies. We found that successful $\mathrm{H}$. pylori eradication induced platelet response in $37.3 \%$ of 83 cases included in our study.
\end{abstract} Keywords: H. pylori, Immune thrombocytopenic purpura, Platelet count.

\section{INTRODUCTION}

Immune thrombocytopenic purpura (ITP), also known as idiopathic thrombocytopenic purpura, is an autoimmune bleeding disorder characterized by bleeding due to isolated thrombocytopenia with a normal bone marrow in the absence of other causes of low platelets ${ }^{(\mathbf{1}, 2)}$. It causes a characteristic red or purple bruise-like rash and an increased tendency to bleed ${ }^{(3)}$.

The exact mechanism of autoimmunity leading to ITP is still unclear. Under non-pathological conditions the immune system is finely regulated by humoral and cellular components, including primarily regulatory $\mathrm{T}$ and $B$ cells ( $T$ regs and $B$ regs). Alteration of the balance between these regulatory cells and effectors results in a breakdown of the immune tolerance causing increased platelet clearance and impaired thrombopoiesis. Similarly to other autoimmune disorders, molecular mimicry with bacterial or viral proteins might be one reason for pathogenesis of ITP. In fact, it was reported a cross-reactivity of anti-platelet autoantibodies with human immunodeficiency virus, hepatitis $\mathrm{C}$ virus and Helicobacter pylori in secondary ITP ${ }^{(\mathbf{1})}$.

Helicobacter pylori (H. pylori), previously known as Campylobacter pylori, is a gram-negative, helicallyshaped, microaerophilic bacterium usually found in the stomach ${ }^{(4)}$. Its helical shape (from which the genus name, helicobacter, derives) is thought to have evolved in order to penetrate the mucoid lining of the stomach and thereby establish infection and causes mainly chronic gastritis and gastric ulcer ${ }^{(5)}$.

Many investigators have proposed causal associations between $\mathrm{H}$. pylori and a wide range of other diseases, e.g. idiopathic thrombocytopenic purpura, iron deficiency anemia, atherosclerosis, and Alzheimer's disease $^{(\boldsymbol{6})}$.

Aim of the work was to detect the association of H. pylori infection and idiopathic thrombocytopenic purpura in children and the effect of eradication of the infection on platelet count in these patients.

\section{PATIENTS AND METHODS}

This study was conducted on 83 chronic idiopathic thrombocytopenic purpura children with age range from 3 to 13 years. They were 46 males 37 females. Also the study included 104 healthy individual with the same range of age. They were 58 males and 46 females as a control group. This was between October 2018 and September 2019 in the Pediatric Hematology and GIT Clinics or inpatients wards of Pediatric Department, in Sohag University Hospital.

Inclusion Criteria: The patients were children aged less than 14 years who were diagnosed with chronic idiopathic thrombocytopenic purpura with no other recognized cause of thrombocytopenia, such as infection, systemic lupus erythematosus, myeloproliferative or lymphoproliferative disorders.

Exclusion Criteria: All children who were diagnosed thrombocytopenic purpura as a part of systemic disease or infection and children with age older than 14 years.

We chose group of children who were diagnosed ITP and stopped any treatment they were receiving it (patient group) and group of children with normal count of platelet (control group) then both were investigated for $\mathrm{H}$. pylori by non-invasive method, which was This article is an open access article distributed under the terms and conditions of the Creative Commons Attribution (CC BY-SA) license (http://creativecommons.org/licenses/by/4.0/) 
detection of antigen in stool. This test is considered to be one of the most accurate tests with both sensitivity and specificity in the range of $90 \%$ to $95 \%$.

Then according to the result of the test each group were divided into two groups:

Control group: included group of $+\mathrm{ve} \mathrm{H}$. pylori and another one -ve $\mathrm{H}$.pylori.

Patient group: was divided into:

Group 1: ITP children with-ve H. Pylori.

This group received the ordinary treatment of ITP

Group 2: ITP children with +ve H. Pylori.

This group received complete course of $\mathrm{H}$. pylori treatment (proton pump inhibitor, clarithromycin, and amoxicillin) then after 6-8 weeks of end of treatment, this group was reinvestigated for $\mathrm{H}$. pylori antigen in stool, and according to the result was subdivided into:

- Group A : who was successfully eradicated of H. pylori infection so the another $\mathrm{H}$. pylori antigen in stool was -ve.

- Group B : who wasn't successfully eradicated of H. pylori infection and the other $\mathrm{H}$. pylori test was +ve.

Then all groups of patients were checked for number of platelet by C.B.C. Then we evaluated the effect of eradication of $\mathrm{H}$. pylori infection on platelet recovery.

Ethical consideration: An informed consent was taken from caregivers of patients included in the study and the protocol of the study was approved by Ethics Scientific Committee at Sohag Faculty of Medicine.
All patient data, measurement and outcomes was confidential and was collected in a special case report formula.

\section{Statistical analysis}

Recorded data were analyzed using the statistical package for the social sciences, version 20.0 (SPSS Inc., Chicago, Illinois, USA). Quantitative data were expressed as mean \pm standard deviation (SD), and median. Qualitative data were expressed as frequency and percentage. Independent-samples t-test of significance was used when comparing between two means. Chi-square $\left(\mathrm{x}^{2}\right)$ test or Fisher exact test of significance was used in order to compare proportions between two qualitative parameters. The confidence interval was set to $95 \%$ and the margin of error accepted was set to $5 \%$. The p-value was considered significant as the following: $\mathrm{P}$-value $<0.05$ was considered significant. P-value $<0.001$ was considered as highly significant. P-value $>0.05$ was considered insignificant.

\section{RESULTS}

The demographic data of the patients group and control group are shown in table 1 . The BMI of patient group ranged from 19 to $25 \%$ while in control group from 19 to $26 \%$. The platelets count of studied groups ranged from 9 to 112 while in control group from 198 to 495 .

Table (1): Comparison between patients and control groups regarding demographic characteristics at the beginning of the study

\begin{tabular}{|c|c|c|c|}
\hline \multirow[t]{3}{*}{ Parameters } & \multicolumn{2}{|c|}{ Groups } & \multirow[t]{3}{*}{$\mathbf{P}$} \\
\hline & Patient group & Control group & \\
\hline & $\mathrm{N}=83(\%)$ & $\mathrm{N}=104(\%)$ & \\
\hline $\begin{array}{l}\text { Gender: } \\
\text { Female } \\
\text { Male }\end{array}$ & $\begin{array}{l}37(44.6) \\
46(55.4)\end{array}$ & $\begin{array}{l}46(44.2) \\
58(55.8)\end{array}$ & 0.962 \\
\hline $\begin{array}{l}\text { Age (years) } \\
\text { Mean } \pm \text { SD } \\
\text { Range }\end{array}$ & $\begin{array}{c}8.28 \pm 2.41 \\
3-13\end{array}$ & $\begin{array}{c}8.38 \pm 2.6 \\
3-13\end{array}$ & 0.772 \\
\hline $\begin{array}{l}\text { BMI: } \\
\text { Mean } \pm \text { SD }\end{array}$ & $22.02 \pm 1.5$ & $22.84 \pm 1.55$ & $<0.001^{* *}$ \\
\hline $\begin{array}{l}\text { Platelet count: } \\
\text { Mean } \pm \text { SD } \\
\text { Median }\end{array}$ & $\begin{array}{c}57.46 \pm 2.78 \\
61\end{array}$ & $\begin{array}{c}321.63 \pm 6.78 \\
322.5\end{array}$ & $<0.001 * *$ \\
\hline
\end{tabular}

$* * p \leq 0.001$ is statistically highly significant

The prevalence of $\mathrm{H}$. pylori among patients group was markedly higher than control group (Table 2).

Table (2): Comparison between patients and control groups regarding $H$. pylori antigen in stool

\begin{tabular}{|l|c|c|c|c|}
\hline \multirow{2}{*}{ H. pylori antigen in stool } & \multicolumn{2}{|c|}{ Groups } & \multirow{2}{*}{ P } & \multirow{2}{*}{ OR 95\% CI) } \\
\cline { 2 - 3 } & Patient group & Control group & & \\
\cline { 2 - 3 } & $\mathbf{N = 8 3 ( \% )}$ & $\mathbf{N = 1 0 4}(\mathbf{\%})$ & & \\
\hline At the start: & $28(23.8)$ & $73(70.2)$ & \multirow{2}{*}{$<0.001^{* *}$} & 4.63 \\
Negative & $55(66.2)$ & $31(29.8)$ & & $(2.49-8.59)$ \\
Positive & & & \\
\hline
\end{tabular}

OR Odds ratio CI Confidence Interval, ${ }^{* *} \mathrm{p} \leq 0.001$ is statistically highly

47 cases out of $55 \mathrm{H}$. pylori positive patients became negative after receiving the triple therapy, while twenty nine cases of control became negative and two cases still had positive one (table 3 ). 
Table (3): Comparison between patients and control groups regarding $\mathbf{H}$. pylori antigen in stool after treatment

\begin{tabular}{|l|c|c|c|}
\hline \multirow{2}{*}{ H. pylori antigen in stool } & \multicolumn{2}{|c|}{ Groups } & \multirow{2}{*}{ P } \\
\cline { 2 - 3 } & Patient group & Control group & \multirow{2}{*}{} \\
\cline { 2 - 3 } & $\mathbf{N = 5 5 ( \% )}$ & $\mathbf{N = 3 1}(\boldsymbol{\%})$ & \\
\hline *After triple therapy : & $47(85.4)$ & $29(98.1)$ & \multirow{2}{*}{0.318} \\
Negative & $8(14.6)$ & $2(1.9)$ & \\
Positive & &
\end{tabular}

*Triple therapy: proton pump inhibitor, clarithromycin, amoxicillin.

There were no differences between positive and negative H. pylori ITP groups after eradication therapy regarding gender, age, disease duration, GIT symptoms, or baseline platelet count. On the other hand there was significant difference in BMI of groups (all patients with seroconversion had higher BMI) (Table 4).

Table (4): Comparison between the studied groups (ITP patients with positive and negative H. pylori antigen in stool after eradication therapy) regarding demographic and disease specific data

\begin{tabular}{|l|c|c|c|c|}
\hline \multirow{2}{*}{ Parameters } & \multicolumn{2}{|c|}{ H. pylori stool antigen } & Total & \multirow{2}{*}{ P } \\
\cline { 2 - 3 } & Negative & Positive & N=55(\%) & \\
\cline { 2 - 3 } Gender: & N=47(\%) & N=8(\%) & & \\
Female & & & & \\
Male & $20(86.9)$ & $3(13.1)$ & $23(36.4)$ & 0.999 \\
\hline Age: & $27(84.3)$ & $5(15.7)$ & $32(63.6)$ & \\
Mean \pm SD & & & & \\
\hline BMI: & $8.09 \pm 1.7$ & $7.13 \pm 2.64$ & $8.28 \pm 2.41$ & 0.244 \\
Mean \pm SD & & & & \\
\hline Baseline Platelet & $22.03 \pm 1.79$ & $21.13 \pm 0.83$ & $22.02 \pm 1.5$ & 0.171 \\
count: & & & & \\
Mean \pm SD & $64.7 \pm 7.46$ & $60.75 \pm 8.16$ & $57.46 \pm 2.78$ & 0.338 \\
\hline Duration: & & & & \\
Mean \pm SD & $1.28 \pm 0.53$ & $1.13 \pm 0.56$ & $1.14 \pm 0.55$ & 0.479 \\
\hline $\begin{array}{l}\text { GI symptoms: } \\
\text { No }\end{array}$ & $33(70.2)$ & $8(29,8)$ & $41(74.5)$ & \\
Abdominal pain & $3(100)$ & $0(0)$ & $3(5.4)$ & 0.430 \\
Epigastric pain & $4(100)$ & $0(0)$ & $4(7.2)$ & \\
Gastritis & $5(100)$ & $0(0)$ & $5(9)$ & \\
\hline
\end{tabular}

There was significant increase in platelet count in the group with $\mathrm{H}$. pylori negative after triple therapy,

while there was no-significant change in it in $\mathrm{H}$. pylori positive group. There was no significant difference between the studied groups regarding baseline platelet count and after 1 month. While there was significant increase in platelet count in successfully eradicated group comparing to non-eradicated one at 6 months (Table 5).

Table (5): Comparison between successfully eradicated ITP patients and non- eradicated as regard platelet count after 1 and 6 months

\begin{tabular}{|l|c|c|c|}
\hline \multirow{2}{*}{ Platelet count } & \multicolumn{2}{|c|}{ Group } & \multirow{2}{*}{ P } \\
\cline { 2 - 3 } & H. pylori negative $(\mathbf{n}=\mathbf{4 7})$ & H. pylori positive $(\mathbf{n}=\mathbf{8})$ & \\
\cline { 2 - 3 } Baseline & Mean \pm SD & Mean \pm SD & 0.338 \\
\hline At 1 month & $64.7 \pm 17.46$ & $60.75 \pm 18.16$ & 0.311 \\
\hline At 6 months & $76.65 \pm 16.57$ & $65 \pm 12.6$ & $0.04 *$ \\
\hline P $($ F) & $95.57 \pm 29.85$ & $70 \pm 31.61$ & \\
\hline
\end{tabular}

${ }^{*} p<0.05$ is statistically significant ${ }^{* *} p \leq 0.001$ is statistically highly significant $t$ Independent sample t test $F$

There was statistically significant platelet response in negative H. pylori-ITP patient after eradication therapy by 1 and 6 months (Table 6). 
Table (6): Relation between ITP patients with successfully eradication for $\mathrm{H}$. pylori infection and with nonsuccessfully eradication regarding platelet response

\begin{tabular}{|c|c|c|c|}
\hline \multirow{3}{*}{$\begin{array}{l}\text { Platelet count } \\
\text { response }\end{array}$} & \multicolumn{2}{|c|}{ Group } & \multirow[t]{3}{*}{$\mathbf{P}$} \\
\hline & H. pylori negative $(n=47)$ & H. pylori positive $(n=8)$ & \\
\hline & $\mathbf{N}(\%)$ & $\mathbf{N}(\%)$ & \\
\hline $\begin{array}{l}\text { At } 1 \text { month: } \\
\text { NR* } \\
\text { PR* }\end{array}$ & $\begin{array}{l}27(57.4) \\
20(42.6)\end{array}$ & $\begin{array}{l}7(87.5) \\
1(12.5)\end{array}$ & 0.136 \\
\hline $\begin{array}{l}\text { At } 6 \text { months: } \\
\text { NR } \\
\text { PR } \\
\text { CR* }\end{array}$ & $\begin{array}{c}16(34) \\
25(53.5) \\
6(12.7)\end{array}$ & $\begin{array}{c}7(87.5) \\
1(12.5) \\
0(0)\end{array}$ & $0.017 *$ \\
\hline
\end{tabular}

$* p<0.05$ is statistically significant, *NR (no response): no improvement in platelet count, *PR (partial response): improvement in platelet count but not exceed $150 \times 10^{9} / \mathrm{L}, * C R$ (complete response): platelet count exceed 150 $\times 10^{9} / L$.

we found a significant difference in the number of responders to eradication therapy; 31 cases showed increase in platelet count, while only 8 cases of $\mathrm{H}$. pylori negative had spontaneous remission. In addition to that none of them showed complete response (Table 7).

Table (7): Relation between ITP patients with successfully eradication and ITP patients with negative H. pylori test from the start regarding platelet response

\begin{tabular}{|l|c|c|c|}
\hline \multirow{2}{*}{ latelet count response } & \multicolumn{2}{|c|}{ Group } & \multirow{2}{*}{ P } \\
\cline { 2 - 3 } & H. pylori negative (n=47) & H. pylori negative (n=28) & \\
\hline At 1 month: & $\mathbf{N}(\%)$ & $\mathbf{N}(\mathbf{\%})$ & \multirow{2}{*}{0.081} \\
NR* & $27(57.4)$ & $22(71.5)$ & \\
PR* & $20(42.6)$ & $6(21.4)$ & \\
\hline At 6 months: & & & \\
NR & $16(34)$ & $20(71.5)$ & $<0.004 *$ \\
PR & $25(53.5)$ & $8(28.5)$ & \\
CR* & $6(12.7)$ & $0(0)$ & \\
\hline
\end{tabular}

$*_{p}<0.05$ is statistically significant, *NR (no response): no improvement in platelet count, *PR (partial response): improvement in platelet count but not exceed $150 \times 10^{9} / \mathrm{L}, * C R$ (complete response) : platelet count exceed 150 $\times 10^{9} / L$.

\section{DISCUSSION}

We have studied 83 ITP patients; their age ranged from 3 to 13 years. Also the study included control group of 104 cases; their age ranged from 3 to 13 years. This age range was similar to the one at Abdollahi et al. ${ }^{(7)}$, Veronica et al. ${ }^{(8)}$ and Loffredo et al. ${ }^{(9)}$ studies.

The male percentage included in this study in patients group was $55.4 \%$ that was higher compared with the patients who took part in Abdollahi et al. ${ }^{(7)}$, Veronica et al. ${ }^{\left({ }^{(8)}\right.}$ and Loffredo et al. ${ }^{(9)}$ studies which was $33.4 \%, 383 \%, 33.3 \%$ respectively, while the females in our study were $44.6 \%$. The male percentage in our control group was $55.8 \%$ while the females were $44.2 \%$. The higher male percentage was because the study was done in developing country.

The mean body mass index (BMI) of patient group was lower than that of control group. This could be explained by presence of chronic illness (chronic ITP) or due to long courses of steroid administration.

In our study we chose patients with platelet count less than $150 \times 10^{9} / \mathrm{L}$ persisting for more than six months and this was similar to Loffredo et al. ${ }^{(9)}$ study, while the cases in Veronica et al. ${ }^{\left({ }^{(8)}\right.}$ and Russo et al. ${ }^{\left({ }^{(10)}\right.}$ studies showed platelet count less than $100 \times 10^{9} / \mathrm{L}$ and persisting for 12 months.

In this study 83 cases of patients group and 104 cases of control group were screened for $\mathrm{H}$. pylori infection which could be diagnosed by invasive methods like endoscopy, mucus biopsy of the stomach and Giemsa stain. Noninvasive methods applied to that effect include urease test and culture. Each method of diagnosis has its own advantages and disadvantages. The urease test or Ag detection are more accurate with 90-95\% sensitivity and specificity. The methods of measuring Abs of $\mathrm{H}$. pylori may be less costly, but they are less accurate with 50-95\% sensitivity and specificity. Therefore in this study, the detection of $\mathrm{H}$. pylori antigen in stool method was applied on our groups. Also this method was the same used one in Abdollahi et al. ${ }^{(7)}$, Russo et al. ${ }^{(10)}$, Loffredo et al. ${ }^{(9)}$ and Veronica et al. ${ }^{(8)}$ studies. The prevalence of $\mathrm{H}$. pylori infection among patients groups in these studies were $20 \%, 90.47 \%, 20.5 \%, 6.4 \%$ respectively while in our study it was 55 cases $(66.2 \%)$ in patients group and 31 cases (29.8\%) among control group. These differences likely reflect the variation in the prevalence of $\mathrm{H}$. pylori infection in the general populations of 
different countries, which is higher in developing countries and lower in developed countries. Previous reports in children with chronic idiopathic thrombocytopenia showed a different prevalence rate of $\mathrm{H}$. pylori infection among countries, ranging from $0 \%$ in Finland to $6.4 \%$ in the Netherlands, $12.9 \%$ in Iran, $20 \%$ in Japan, $20.5 \%$ in Italy $29.1 \%$ in Thailand, $31.4 \%$ in Turkey, and $40.9 \%$ in Taiwan.

In this study we found that the prevalence of $\mathrm{H}$. pylori infection among patients group was significantly higher than control group. This result was similar to the one in Abdollahi et al. (7) study; the percentage in patient group was (90.47\%) while in control group was $28.12 \%$. This result supported the association between H. pylori infection and ITP.

We found no difference in gender, age, disease duration or BMI between positive and negative $\mathrm{H}$. pylori ITP patients. Furthermore most of patients group with $\mathrm{H}$. pylori positive test showed asymptomatic $\mathrm{H}$. pylori infection but there were 12 cases $(21.8 \%)$ of them had GIT symptoms like abdominal pain and epigastric pain. This was the same at Abdollahi et al. ${ }^{(7)}$ and Veronica et al. ${ }^{(8)}$ studies.

All cases that had $\mathrm{H}$. pylori positive test were treated with a combination of amoxicillin $50 \mathrm{mg} / \mathrm{kg} / \mathrm{d}$, clarithromycin $10 \mathrm{mg} / \mathrm{kg} / \mathrm{d}$, and omeprazol $1 \mathrm{mg} / \mathrm{kg} / \mathrm{d}$ for two weeks. This protocol of treatment was like ones used in the other studies with slight differences; in Veronica et al. ${ }^{(8)}$ study the protocol was a combination of amoxicillin $15 \mathrm{mg} / \mathrm{kg}$, clarithromycin $7.5 \mathrm{mg} / \mathrm{kg}$ bd. and omeprazol $2 \mathrm{mg} / \mathrm{kg}$ od. for two weeks. While Loffredo et al. ${ }^{\left({ }^{(9)}\right.}$ used a combination of amoxicillin at $50 \mathrm{mg} / \mathrm{kg}$ per day and clarithromycin at $10 \mathrm{mg} / \mathrm{kg}$ per day for 14 days, and omeprazole at $1 \mathrm{mg} / \mathrm{kg}$ per day for 4 weeks. On the other hand Russo et al. ${ }^{(10)}$ used a different one; the first-line protocol for $\mathrm{H}$. pylori eradication was amoxicillin (twice daily for 1 week, $50 \mathrm{mg} / \mathrm{kg} /$ day), clarithromycin (twice daily for 1 week, $15 \mathrm{mg} / \mathrm{kg} /$ day), and omeprazole ( $10 \mathrm{mg} /$ day for patients $<6$ years old, $20 \mathrm{mg} /$ day for patients $>6$ years old; once daily for 2 weeks). Patients who were still $\mathrm{H}$. pyloripositive underwent a second-line eradication cycle, which included amoxicillin and omeprazole at the same doses and schedule as the first-line protocol in addition to metronidazole (three times daily for 1 week, $15 \mathrm{mg} / \mathrm{kg} / \mathrm{day})$.

Eradication success was assessed 6 weeks after the end of treatment using the stool antigen test; 47 cases $(85.4 \%)$ were successfully treated and showed negative result while the other 8 cases of the patients group showed positive test. In the study of Russo et al. (10) the eradication of H. pylori infection was successful in 33/37 patients $(89.2 \%$ ), on the other hand all cases of control group in our study showed negative result after treatment except 2 cases who had positive test.

No differences were found between patients who experienced successful eradication and those who did not in regards to age, gender, duration of disease, GI symptoms, and the basal PLT count. These results were similar to Abdollahi et al. ${ }^{(7)}$ and Russo et al. ${ }^{(10)}$.

In our study, platelet counts were determined after one month of successful eradication of $\mathrm{H}$. pylori infection of patients group and then after 6 months. Eradication was defined as a complete response if the PLT count was greater than $150 \times 10^{9} / \mathrm{L}$ and a partial response if it was at least $50 \times 10^{9} / \mathrm{L}$ with an increase of more than $30 \times 10^{9} / \mathrm{L}$ over the basal count. No response was defined as a PLT count of less than $50 \times 10^{9} / \mathrm{L}$, a lack of a more than $30 \times 10^{9} / \mathrm{L}$ increase, or the need for maintenance therapy. These values were the same at Abdollahi et al. ${ }^{(7)}$, Russo et al. ${ }^{(10)}$ and Veronica et al. (8).

After one month the 47 cases who were successfully treated from $\mathrm{H}$. pylori infection were tested for platelet count were 20 cases $(46.6 \%)$ had partial response, while 27 cases $(57.4 \%$ ) had no response. On the other hand the 8 cases with non-successfully eradication; $7(87.5 \%)$ cases of them showed no response and $1(12.5 \%)$ case showed partial response.

Then after 6 months there was marked increase in platelet count in successfully treated group; 25 cases (53.5\%) group showed partial response, 6 cases (12.7 $\%)$ showed complete response, and 16 cases (34\%) showed no response. While in non-successfully treated group there was no change in platelet response; $87.5 \%$ of them still had no response and $12.5 \%$ still had partial response. Furthermore none of them showed complete platelet response. So there was no significant response in platelet count in non-successfully treated group, which was in agreement with previous reports showing that an ITP response occurs only among patients with successful eradication and not among those with unsuccessful $\mathrm{H}$. pylori eradication ${ }^{(7,10)}$. However these results were against to the results that were reported in Veronica et al. ${ }^{(8)}$ and Loffredo et al. ${ }^{(9)}$ who showed no improvement in platelet count in successfully treated ITP patients.

A comparison of demographic characteristics of responders and non-responders of our study revealed no differences in regard to sex, age, duration of disease, and basal platelet count. Also there were no differences between complete and partial responders in regard to gender, age, age at diagnosis, duration of disease, and basal platelet count. This result was similar to Abdollahi et al. ${ }^{(7)}$ and Russo et al. ${ }^{(10)}$.

On the other hand in comparison between successfully eradicated ITP patients and negative ITP patients from the start regarding platelet response. we found a significant difference in the percentage of responders to eradication therapy; 31 cases out of 55 $(65.5 \%)$ of successfully eradicated ITP patients $(25$ partial response and 6 with complete response) showed increase in platelet count, while 8 cases out of 28 $(28.5 \%)$ of $\mathrm{H}$. pylori negative showed partial response of platelet count. Furthermore, none of them showed complete response, which was in agreement with 
previous report by Russo et al. ${ }^{(10)}$. The percentage of response can be said in other words; the number of responders (had increased platelet count) to eradication was $(37.5 \%)$ of total patients group and $(9.5 \%)$ had spontaneous remission.

The higher prevalence of $\mathrm{H}$. pylori infection among cITP children and the significant increase in platelet count after $\mathrm{H}$. pylori eradication suggests a role of $\mathrm{H}$. pylori in cITP disease. Maybe H. pylori infection does not play a primary role in the onset and /or persistence of ITP, but it may have an additional effect on the thrombocytopenia in ITP while not being the sole cause.

Clinical benefit occurred in 31 cases of the 83 cITP patients screened; nevertheless, we have a simple, inexpensive, non-invasive screening method; the adverse events of eradication therapy are negligible, together with an affordable cost, all uncommon attributes among the specific treatments currently available for cITP; therefore it is worthwhile to test for and treat $\mathrm{H}$. pylori infection.

\section{CONCLUSION}

Our data showed a prevalence of $\mathrm{H}$. pylori infection in cITP pediatrics that was similar to other studies. We found that successful $\mathrm{H}$. pylori eradication induced platelet response in $37.3 \%$ of 83 cases included in our study.

Even if a causative role for $\mathrm{H}$. pylori infection cannot be demonstrated, the availability of a safe, tolerable, and in expensive method for detecting and treating $\mathrm{H}$. pylori suggests that it may be appropriate to investigate and eradicate $\mathrm{H}$. pylori infection in cITP children.

\section{REFERENCES}

1. Marini I, Bakchoul T (2019): Pathophysiology of autoimmune thrombocytopenia: current insight with a focus on thrombopoiesis. Hamostaseologie, 39(3):227237.

2. Rodeghiero F, Stasi R, Gernsheimer T et al. (2009): Standardization of terminology, definitions and outcome criteria in immune thrombocytopenic purpura of adults and children: report from an international working group. Blood, 113(11):2386-93.

3. Matzdorff A, Meyer O, Ostermann H et al. (2018): Immune thrombocytopenia - current diagnostics and therapy. Oncol Res Treat., 41(5):1-30.

4. Alfarouk K, Bashir A, Aljarbou A et al. (2019): The Possible role of helicobacter pylori in gastric cancer and its management. Frontiers in Oncology, 9: 75-81.

5. Yamaoka Y (2008): Helicobacter pylori: Molecular Genetics and Cellular Biology. Caister Academic Press, Pp. 262. https://www.caister.com/hpl2

6. Laird-Fick H, Saini S, Hillard J (2016): Gastric adenocarcinoma: the role of Helicobacter pylori in pathogenesis and prevention efforts. Postgraduate Medical Journal, 92: 471-7.

7. Abdollahi A, Shoar S, Ghasemi S et al. (2015): Is Helicobacter pylori infection a risk factor for idiopathic thrombocytopenic purpura in children? Ann Afr Med., 14:177-181.

8. Veronica M, Harriet H, Rienk Y (2007): H. pylori infection in childhood chronic immune thrombocytopenic purpura. Haematologica, 92: 570-5.

9. Loffredo G, Marzano M, Migliorat R et al. (2007): The relationship between immune thrombocytopenic purpura and Helicopacter pylori infection in children: where is the truth ? Eur J Pediatr., 166:1067-1068.

10. Russo G, Mraglia V, Branciforte F et al. (2010): Effect of eradication of Helicobacter pylori in children with chronic thrombocytopenia, Pediatric Blood \& Cancer, 56(2):273-278. 